\title{
Novel acyl sulfonamide LY573636-sodium: Effect on hematopoietic malignant cells
}

\author{
TALIN HARITUNIANS ${ }^{1 *}$, SASKIA GUELLER $^{1 *}$, JAMES O'KELLY $^{1}$, \\ ROBERT ILARIA $\mathrm{Jr}^{2}$ and H. PHILLIP KOEFFLER ${ }^{1}$ \\ ${ }^{1}$ Division of Hematology/Oncology, Cedars-Sinai Medical Center and UCLA \\ School of Medicine, 8700 Beverly Blvd., Los Angeles, CA 90048; ${ }^{2}$ Lilly Research \\ Laboratories, Lilly Corporate Center DC 2133, Indianapolis, IN 46285, USA
}

Received April 22, 2008; Accepted May 26, 2008

DOI: 10.3892/or_00000135

\begin{abstract}
LY573636-sodium is a promising anti-tumor agent, which causes growth arrest and apoptosis of a variety of human solid tumors in vitro and in vivo. Moreover, studies have shown that the compound is selectively toxic towards tumor cells over their normal counterparts. This targeted effect makes LY573636 a candidate for combined therapy regimens in patients with advanced or resistant cancers. We studied for the first time, the anti-tumor properties of LY573636 against a variety of human hematopoietic malignancies, including AML, B-ALL, large B-cell and mantle cell lymphoma cell lines. Cells were treated with the compound in vitro and its effect on cell proliferation, apoptosis and differentiation was determined. The cell lines underwent growth arrest in response to treatment with LY573636 in a dose-dependent manner. This antiproliferative activity was associated with the induction of apoptosis, loss of mitochondrial membrane potential and induction of reactive oxygen species. Furthermore, we showed that LY573636 was able to induce granulocytic/monocytic differentiation of HL60 and U937 cells. LY573636, as shown before in solid tumors, is effective in hematopoietic cell lines as well. These data suggest the use of LY573636 alone or in combination with conventional chemotherapeutic regimens in hematopoietic malignancies.
\end{abstract}

\section{Introduction}

LY573636-sodium (hereafter referred to as LY573636), a benzamide, N-[(5-bromo-2-thienyl)sulfonyl]-2,4-dichloro-

Correspondence to: Dr Saskia Gueller, Division of Hematology/ Oncology, Cedars-Sinai Medical Center and UCLA School of Medicine, 8700 Beverly Blvd., Los Angeles, CA 90048, USA

E-mail: sgueller@gmail.com

${ }^{*}$ Contributed equally

Key words: small-molecule drugs, acyl sulfonamides, LY573636, hematopoietic malignancies sodium salt, was initially identified for its selective activity against solid tumors using an in vitro soft agar disk diffusion assay. This compound has demonstrated significant dosedependent antitumor activity against xenograft models of HCT116 and SW620 human colon cancers, as well as A375 melanoma (1; Lilly Research Laboratories, LRL, unpublished data). Other acyl sulfonamide analogues identified during the development of LY573636 have been reported in recent years to exhibit considerable antitumor activity, particularly against human colon, lung, prostate, ovarian and breast xenografts growing in immunodeficient mice $(2,3)$. In addition, the toxicity of LY573636 has been evaluated in vitro and in vivo in different species including rats and beagle dogs (LRL, unpublished data). Currently, a phase I study of LY573636 has been completed, with bone marrow suppression, most commonly thrombocytopenia, identified as the dose-limiting toxicity. Phase II studies in non-small cell lung cancer, malignant metastatic melanoma, ovarian cancer and soft tissue sarcoma are currently in progress (4). Importantly, the National Cancer Institute's COMPARE analysis did not identify any similarities between LY573636 and any known cytotoxic compound suggesting a unique mechanism of action. These findings are consistent with recent pre-clinical mechanism of action studies indicating that LY573636-induced apoptosis occurs by a mitochondrialtargeted mechanism (LRL, unpublished data). To date, no one has evaluated the effects of LY573636 against malignant hematopoietic cells; in this study, we examined the antileukemic and anti-lymphoma activity of the compound using a large variety of cell lines.

\section{Materials and methods}

Cell culture. B-cell acute lymphocytic leukemia ( $\mathrm{RCH}$, kindly provided by Dr Janet Rowley, University of Chicago; Reh, a generous gift from Dr Gary Gilliland, Harvard University; BALL-1, kindly provided by Dr Sven de Vos, University of California, Los Angeles), Burkitt's lymphoma [Daudi, American Type Culture Collection (ATCC), Manassas, VA], diffuse large B-cell lymphoma (MD901, kindly provided by Dr Tohru Miki, Tokyo Medical and Dental University, Japan; LY4, generously donated by Ari Melnick, Albert 
Table I. LY573636 inhibits the growth of various human leukemia and lymphoma cell lines. ${ }^{a}$

\begin{tabular}{llr}
\hline Cell lines & \multicolumn{1}{c}{ Cell type } & ED $_{50}(\mu \mathrm{M} \mathrm{LY573636)}$ \\
\hline HL60 & Acute myeloid leukemia & 7 \\
U937 & Acute myeloid leukemia & $>40$ \\
Reh & B-cell acute lymphocytic leukemia & 23 \\
RCH & B-cell acute lymphocytic leukemia & 12 \\
BALL1 & B-cell acute lymphocytic leukemia & 5 \\
MD901 & Diffuse large B-cell lymphoma & 19 \\
LY4 & Diffuse large B-cell lymphoma & 25 \\
Daudi & Burkitt's lymphoma & 31 \\
Jeko1 & Mantle cell lymphoma & 16 \\
SP49 & Mantle cell lymphoma & 21 \\
NCEB1 & Mantle cell lymphoma & 13
\end{tabular}

${ }^{a}$ Cells were cultured for $96 \mathrm{~h}$ with varying concentrations of LY573636 (0.5-40 $\left.\mu \mathrm{M}\right)$ and cell growth was measured by MTT assay. Data were plotted and the effective dose, which inhibited $50 \%$ of growth $\left(\mathrm{ED}_{50}\right)$ was calculated for each cell line. Results represent the mean $\mathrm{ED}_{50}$ of duplicate experiments done with triplicate wells per point.

Einstein College of Medicine) and myeloid leukemia cell lines (HL60 and U937, ATCC) were maintained in RPMI1640 (Invitrogen, Carlsbad, CA), supplemented with 10\% fetal bovine serum (FBS), $10 \mathrm{U} / \mathrm{ml}$ penicillin and $10 \mathrm{mg} / \mathrm{ml}$ streptomycin (P/S) (Invitrogen) at $37^{\circ} \mathrm{C}$ in $5 \% \mathrm{CO}_{2}$. Mantle cell lymphoma cell lines (NCEB1, Jeko1 and SP49) (5) were maintained under similar conditions in RPMI-1640 supplemented with $20 \% \mathrm{FBS}$ and P/S.

MTT proliferation assays. Cells were treated with various concentrations of LY573636 (kindly provided by Lilly Research Laboratories, Indianapolis, IN). 3-(4,5-Dimethylthiazol-2-yl)-2,5-diphenyltetrazolium bromide (MTT) assay (Sigma-Aldrich, St. Louis, MO) was performed, as previously described (6). Briefly, MTT was dissolved in phosphatebuffered saline (PBS) at $5 \mathrm{mg} / \mathrm{ml}$. Approximately 1,000 cells per well were incubated in culture medium for $96 \mathrm{~h}$ in $96-$ well plates then, $10 \mu \mathrm{l}$ of the MTT solution was added. After $4 \mathrm{~h}$ of incubation, $100 \mu 1$ of solubilization solution [20\% sodium dodecyl sulfate (SDS)] was added and the mixture was incubated at $37^{\circ} \mathrm{C}$ for $16 \mathrm{~h}$. In this assay, MTT was cleaved to an orange formazan dye by metabolically active cells and the absorbance of the formazan product was measured with an enzyme-linked immunoabsorbent assay (ELISA) reader at $540 \mathrm{~nm}$.

Apoptosis analyses. Annexin V staining was performed, as previously described (7). Briefly, after a 72-h treatment period with $40 \mu \mathrm{M}$ of LY573636, cells were washed with cold PBS, resuspended in $1 \mathrm{X}$ binding buffer (BD Biosciences, Pharmingen, San Jose, CA) and incubated with fluorescein isothiocyanate (FITC)-conjugated Annexin V and $2.5 \mu \mathrm{g} / \mathrm{ml}$ propidium iodide $(\mathrm{PI})$ at room temperature for $15 \mathrm{~min}$ in the dark. Prior to analysis, $80 \% 1 \mathrm{X}$ binding buffer was added and analysis was performed within $1 \mathrm{~h}$. All studies were performed using the flow cytometry (FACScan, Becton Dickinson, Franklin Lakes, NJ).
Mitochondrial membrane potential. Cells were incubated with either 1,10 or $40 \mu \mathrm{M}$ of LY573636 for $72 \mathrm{~h}$ at $37^{\circ} \mathrm{C}$. The loss of mitochondrial membrane potential was analyzed using the lipophilic cationic dye JC-1 (5,5',6,6' tetrachloro $1,1^{\prime}, 3,3^{\prime}$ tetraethylbenzimidazolylcarbocyanine iodide), according to the manufacturer's protocol (Cell Technology, Mountain View, CA). Briefly, cells were pelleted and resuspended in $1 \mathrm{X} \mathrm{JC}-1$ reagent and incubated for $15 \mathrm{~min}$ at $37^{\circ} \mathrm{C}$. Cells were washed twice with culture media and resuspended in complete media. Analyses were immediately performed by flow cytometry (FACScan).

ROS determination. Cells were incubated with $40 \mu \mathrm{M}$ of LY573636 for either 24,48 or $72 \mathrm{~h}$ at $37^{\circ} \mathrm{C}$. They were washed once with complete media, incubated for $30 \mathrm{~min}$ at $37^{\circ} \mathrm{C}$ with $10 \mu \mathrm{M}$ of DCFDA (2',7' dichlorofluorescein diacetate) (Sigma-Aldrich) in complete media and analyses were immediately performed by flow cytometry (FACScan).

Study of differentiation. Cells were incubated with various concentrations of LY573636 for four days at $37^{\circ} \mathrm{C}$. Cells were washed with phosphate-buffered saline (PBS) and incubated for 30 min with either R phycoerythrin (RPE)-conjugated murine anti-human CD11b or negative control RPE conjugated murine IgG1 antibody (Dako, Carpinteria, CA). The expression of cell surface antigens was determined by flow cytometry (FACScan).

\section{Results}

To determine if LY573636 inhibits the proliferation of malignant hematopoietic cells, different cell lines were cultured in various concentrations of LY573636 $(0.5-40 \mu \mathrm{M})$ for $96 \mathrm{~h}$ and growth inhibition was measured by MTT. The mean \pm SD of duplicate experiments performed in triplicate were plotted and the concentration inhibiting $50 \%$ of growth $\left(\mathrm{ED}_{50}\right)$ was calculated (Table I, with representative dose- 


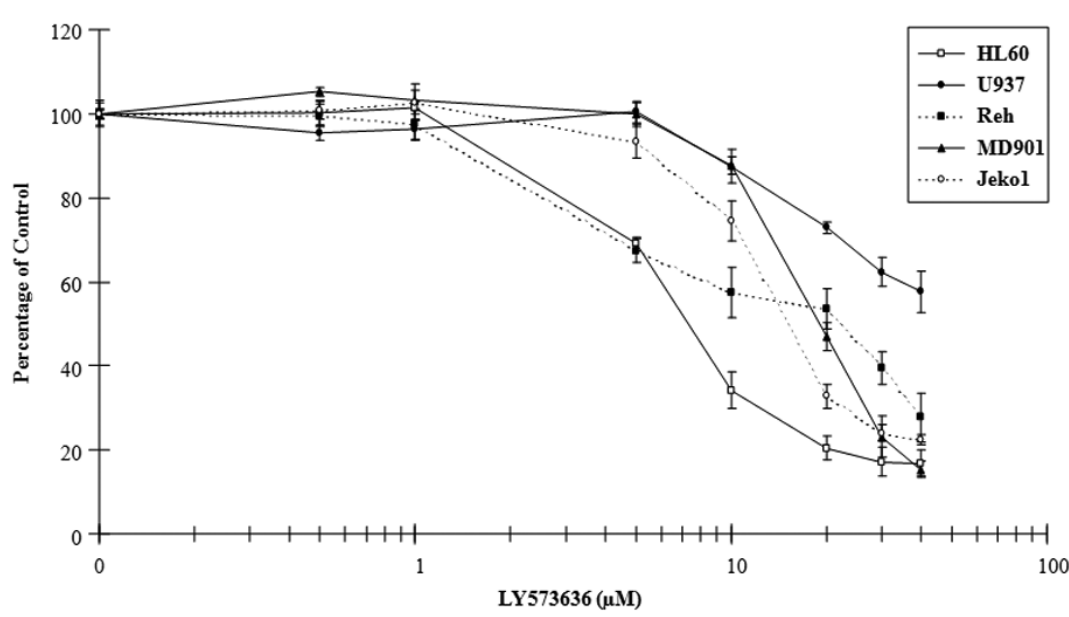

Figure 1. Growth inhibitory activity of LY573636-sodium against HL60, U937, Reh, MD901 and Jeko1 cells. MTT assay measuring the proliferation of

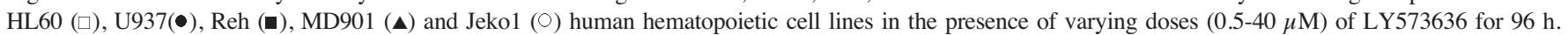
Results for untreated control cells were set at $100 \%$; experimental data are shown as a percentage of the control. Data represent the mean \pm standard error of duplicate experiments performed with triplicate samples per point.

response curves in Fig. 1). In agreement with the antiproliferative effects observed in the solid tumors (LRL, unpublished data), our results demonstrate that LY573636 interfered with the growth potential of a wide panel of human malignant hematopoietic cell lines, including AML $\left(\mathrm{ED}_{50}, 7\right.$ and $>40 \mu \mathrm{M}$ for HL60 and U937 cells, respectively), B-ALL (mean $\mathrm{ED}_{50}, 13 \mu \mathrm{M}$ ), as well as Burkitt's lymphoma, diffuse large B-cell lymphomas and mantle cell lymphomas (mean $\mathrm{ED}_{50}, 21 \mu \mathrm{M}$ ) (Table I). Following this initial screen, we chose to limit the remainder of our studies to a few select cell lines from each subtype: two AML cell lines (HL60 and U937) with disparate $\mathrm{ED}_{50}$ 's, as well as Reh (B-ALL), MD901 (diffuse large B-cell lymphoma) and Jeko1 (mantle cell lymphoma) (Fig. 1).

Previous results demonstrated that LY573636 induced apoptosis in colorectal and ovarian cancer and melanoma cell lines (LRL, unpublished data). To assess whether the antiproliferative effects of LY573636 observed by MTT assay were attributed to an induction of cell death, HL60, U937, Reh, MD901 and Jeko1 cells were cultured in $40 \mu \mathrm{M}$ of LY573636 or control for 24, 48 and $72 \mathrm{~h}$. While no significant effects were evident after 24 and 48 h (data not shown), HL60, Reh and MD901 cells demonstrated between 3.3- to 4.5-fold increase in the percentage of Annexin V- and PI-double positive cells following a 72-h incubation period. Nevertheless, none of the cell lines exhibited $>10 \%$ apoptotic cells (Table II). U937 cells, which were the least sensitive to the growth inhibitory action of LY573636 (Fig. 1, as measured by MTT), still showed an almost 3-fold increase in apoptotic cells in the presence of LY573636 compared to diluent control cells. In contrast, Jeko1 cells did not exhibit any significant induction of apoptosis.

Mitochondrial membrane permeability and the consequential collapse of the electrochemical potential across the mitochondrial membrane are early key steps in the induction of apoptosis and occur through the formation of membrane pores via the activation of pro-apoptotic proteins and release of cytochrome-C into the cytoplasm (8). LY573636-induced
Table II. LY573636 induces apoptosis in HL60, Reh and MD901 cells. ${ }^{a}$

\begin{tabular}{lcc}
\hline \multirow{2}{*}{ Cell lines } & \multicolumn{2}{c}{ Percentage of AnnexinV+/PI+ cells } \\
\cline { 2 - 3 } & Control & LY573636 \\
\hline HL60 & 3 & 10 \\
U937 & 2 & 6 \\
MD901 & 3 & 10 \\
Reh & 2 & 9 \\
Jeko1 & 2 & 2 \\
\hline
\end{tabular}

${ }^{\mathrm{a} C e l l s ~ w e r e ~ c u l t u r e d ~ f o r ~} 72 \mathrm{~h}$ with $40 \mu \mathrm{M}$ of LY573636 and the percentage of Annexin V-and PI-double positive cells was determined by flow cytometry. Data represent the mean of triplicate dishes.

apoptosis in solid tumor cell lines was found to be mediated by the intrinsic, mitochondrial-mediated cell death pathways, associated with a reduction in cellular ATP levels (LRL, unpublished data). To determine whether this mechanism also applied to hematopoietic malignant cell lines used in this study, we looked for changes in mitochondrial membrane potential as a function of mitochondrial damage using the JC-1 dye. In healthy cells, positively charged JC-1 molecules accumulate in aggregates within the negatively charged mitochondria and emit red fluorescence at the $590 \mathrm{~nm}$ spectrum. In apoptotic cells, however, the collapse of the mitochondrial membrane potential interferes with the accumulation of the $\mathrm{JC}-1$ molecules, which remain in the cytoplasm in a monomeric form, emitting green fluorescence at the $527 \mathrm{~nm}$ spectrum. Hence, healthy cells can easily be distinguished from apoptotic cells by their loss of mitochondrial membrane potential.

HL60, U937, Reh, MD901 and Jeko1 cells were cultured with increasing concentrations of LY573636 (1, 10 and $40 \mu \mathrm{M})$ 

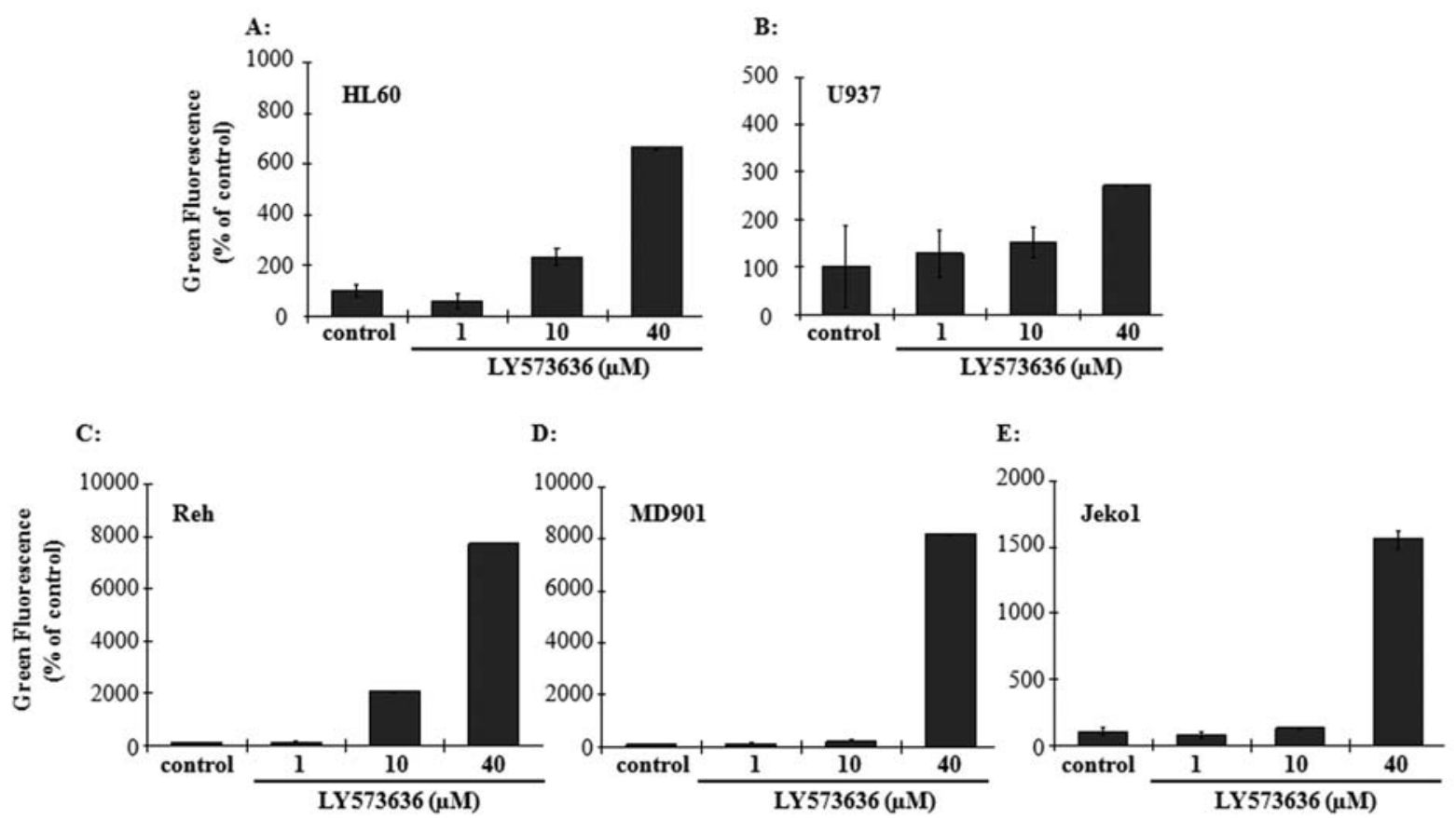

Figure 2. LY573636-sodium induces mitochondrial membrane collapse of HL60, Reh, MD901 and Jeko1 cells. HL60 (A), U937 (B), Reh (C), MD901 (D) and Jeko1 (E) cells were incubated for $72 \mathrm{~h}$ with increasing amounts of LY573636 $(1,10$ or $40 \mu \mathrm{M})$. The loss of mitochondrial membrane potential was determined by measuring changes in the levels of green fluorescence (monomeric JC-1) at the $527 \mathrm{~nm}$ spectrum. Data represent the mean \pm SE of three separate experiments.

A:

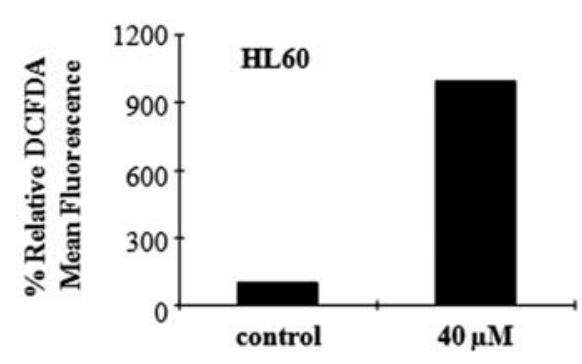

B:

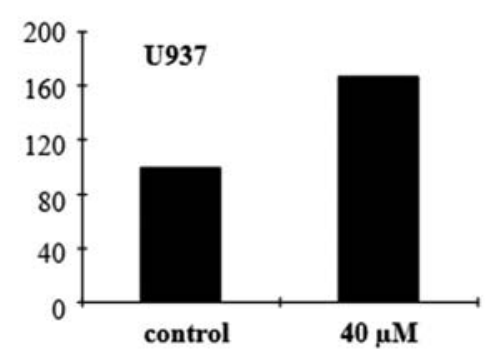

C:

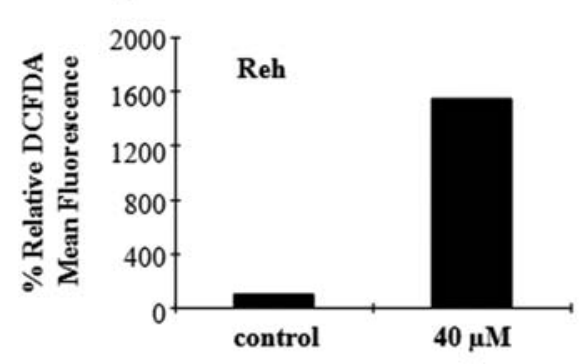

D:

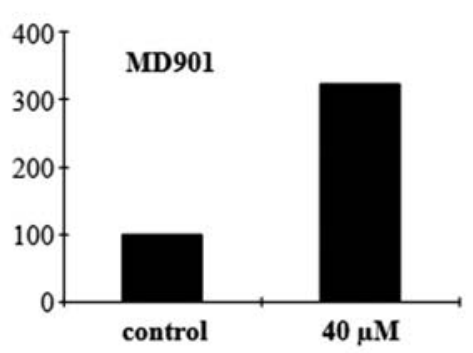

E:

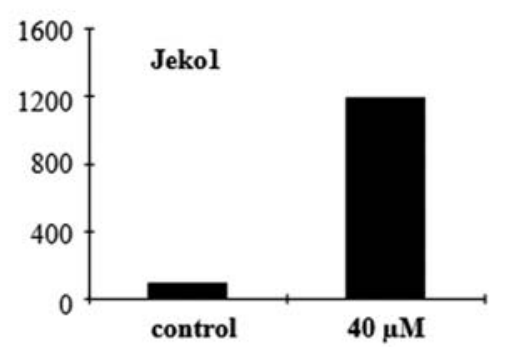

Figure 3. LY573636-sodium induces ROS generation in HL60, Reh, MD901 and Jeko1 cells. HL60 (A), U937 (B), Reh (C), MD901 (D) and Jeko1 (E) cells were cultured for $72 \mathrm{~h}$ with $40 \mu \mathrm{M}$ of LY573636. ROS production was determined by relative mean fluorescence levels of DCFDA. The figure shows data of one representative experiment out of three independent experiments. Results for untreated control cells were set at 100\%, with remaining data shown as a percentage of the control.

for $72 \mathrm{~h}$ (time point at which the induction of apoptosis was observed) and changes in the levels of monomeric JC-1 (green fluorescence) were assessed (Fig. 2). In HL60, Reh and MD901 cells, a dose-dependent increase in green fluorescence, corresponding to a decrease in mitochondrial membrane potential was observed following treatment with LY573636. HL60 cells demonstrated a mean 6.6-fold increase in green fluorescence compared to the control cells (Fig. 2A), while Reh and MD901 exhibited a mean 77- and 81-fold increase, respectively, after culture with $40 \mu \mathrm{M}$ of LY573636 (Fig. 2C 

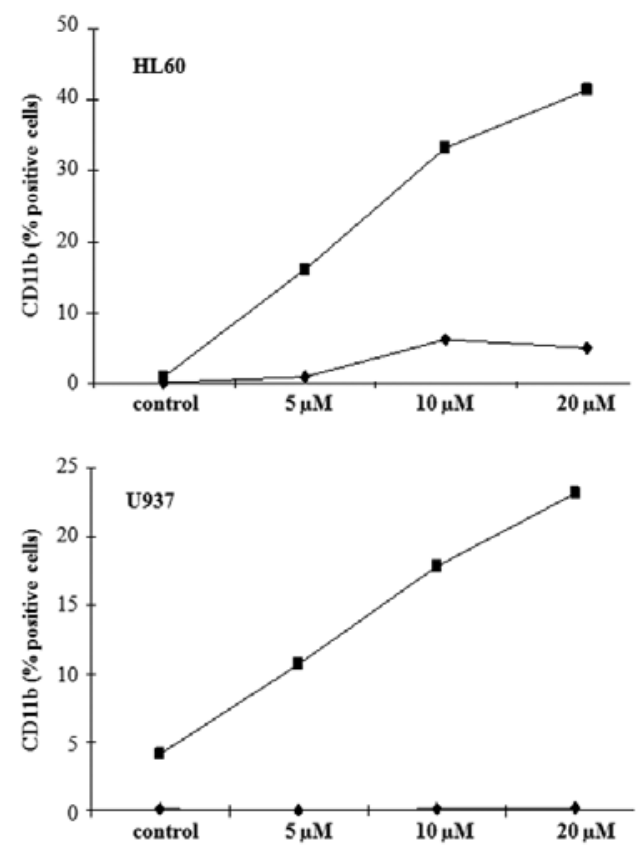

- IgG contro

- CDIlb

Figure 4. LY573636-sodium treatment induces CD11b expression in AML cell lines. Following a 96-h treatment with $20 \mu \mathrm{M}$ of LY573636, HL60 (top) and U937 (bottom) cells were incubated with an RPE-conjugated CD11bspecific antibody ( $\mathbf{(})$ or an RPE-conjugated IgG1 control antibody ( $\bullet$ ) and flow cytometry was performed. Results of each figure are representative of two independent experiments.

and D). Thus, LY573636-induced apoptosis observed in HL60, Reh and MD901 cells coincided with a decrease in their mitochondrial membrane potential. Moreover, U937 cells, which exhibited a lower sensitivity to the antiproliferative (MTT) and pro-apoptotic (Annexin V-positive) activities of LY573636, likewise had a smaller shift toward green fluorescence (only 2.7-fold) (Fig. 2B). Remarkably, although Jeko1 cells did not reveal a significant increase in the percentage of cells undergoing apoptosis, they did, however, demonstrate a 15-fold increase in their levels of green fluorescence (Fig. 2E).

The generation of significant levels of reactive oxygen species (ROS) in a cell occurs as a late event in the induction of apoptosis after the activation of caspases (8). Given our observations of enhanced apoptosis, coupled with the loss of mitochondrial membrane potential in various cell lines, we assessed the levels of ROS production after exposure to LY573636. Following treatment with LY573636 (40 $\mu \mathrm{M} ; 24$, 48, or 72 h), HL60, U937, Reh, MD901 and Jeko1 cells were incubated with DCFDA, which generates fluorescent diclorofluorescein upon oxidation. We observed a significant time-dependent increase in levels of ROS production (measured by relative DCFDA mean fluorescence) in HL60, Reh and Jeko1 cells (>10-fold) (Fig. 3A, C and E). The MD901 cells generated an $\sim 3$-fold rise in ROS levels after $72 \mathrm{~h}$ of treatment (Fig. 3D). Once again, the least sensitive cell line, U937, did not have a significant enhancement in DCFDA fluorescence, with relative levels comparable to the control even after $72 \mathrm{~h}$ of exposure (Fig. 3B). This is consistent with previous findings in HCT116 colon cancer cells that showed a dose-dependent, congruent induction of apoptosis and ROS upon LY573636 treatment (LRL, unpublished data).

Since the induction of differentiation represents a potential therapeutic strategy in AML, we investigated the ability of LY573636 to induce myeloid differentiation. Myeloid leukemic cells, such as HL60 and U937 used in this study, express specific cell surface antigens as they undergo differentiation (9). Accordingly, we evaluated by flow cytometry the ability of LY573636 to induce differentiation as measured by the expression of the monocyte- and granulocyte-marker, CD11b. Following a 4-day exposure to a non-apoptotic dose of LY573636 $(20 \mu \mathrm{M})$, HL60 and U937 exhibited a dose-dependent increase in the percentage of CD11b expressing cells, with $>40 \%$ and $20 \%$ of the cells becoming positive, respectively (Fig. 4).

\section{Discussion}

Acyl sulfonamides, such as LY573636, were initially recognized for their significant antiproliferative activities in solid tumor cell lines, though their mechanism of action was unknown (1). Subsequent studies have revealed that LY573636 induces apoptosis via a mitochondrial-mediated mechanism, which appears unique among other anti-cancer compounds. However, little is known of the effects of LY573636 on hematopoietic malignancies, which prompted our study of these cells.

Consistent with findings in solid tumors (LRL, unpublished data), the antiproliferative effects of LY573636 observed in our studies demonstrate that LY573636 inhibits growth and induces apoptosis in diverse human malignant hematopoietic cell lines. In most cell lines, a dose-dependent decrease of membrane potential and elevated levels of ROS upon treatment of sensitive cell lines was associated with apoptosis. Importantly, these effects were achieved at LY573636 concentrations that ranged clearly below the maximum tolerated targeted Cmax concentration in patients $(420 \mu \mathrm{g} / \mathrm{ml}$ or $960 \mu \mathrm{M}$ ) identified in the phase I study JZAA and taken forward into Phase II (4).

Notably, apoptosis rates upon a 72-h treatment with LY573636 did not exceed $10 \%$ of the cells, though this still demonstrates 3- to 4.5-fold increase of apoptotic cells. Compared to the 8-fold increase in HCT-116 colon cancer cells upon treatment with lower concentrations of LY573636 (LRL, unpublished data), the hematopoietic cells seem to be less sensitive to this compound. Nevertheless, LY57636 alone or in combination might be useful as a therapy for leukemia or lymphoma given the relatively long half-life in humans ( $\sim 11$ days), which can provide prolonged exposure to potentially active drug concentrations (4) (LRL, unpublished data). Furthermore, the compound appears to have a novel mechanism of action supporting the further exploration of LY573636 as a therapeutic agent for acute leukemias and lymphomas.

\section{Acknowledgements}

This study was supported in part by the Sheryl Weissberg Lymphoma Research Foundation (H.P.K.), the Tower Cancer Research Foundation Fellowship (T.H., S.G.), the Deutsche 
Krebshilfe (SG) and a gift from Lilly Research Laboratories. H.P.K. holds the Mark Goodson Chair of Oncology Research at Cedars-Sinai Medical Center and is a member of the Molecular Biology Institute and Jonsson Comprehensive Cancer Center at UCLA.

\section{References}

1. Mader MM: Novel antiproliferative antitumor agents. Curr Opin Drug Discov Devel 8: 613-618, 2005.

2. Corbett TH, White K, Polin L, Kushner J, Paluch J, Shih C and Grossman CS: Discovery and preclinical antitumor efficacy evaluations of LY32262 and LY33169. Invest New Drugs 21: 33-45, 2003.

3. Lobb KL, Hipskind PA, Aikins JA, et al: Acyl sulfonamide anti-proliferatives: benzene substituent structure-activity relationships for a novel class of antitumor agents. J Med Chem 47: 5367-5380, 2004.

4. Ilaria RL Jr, Simon GR, Sovak M, et al: Phase I study of LY573636-sodium, an acylsulfonamide anti-cancer compound with a novel mechanism of action, administered as 2-hour IV infusion in patients with advanced solid tumors. ASCO Annual Meeting Proceedings Part I. J Clin Oncol 25: 2515, 2007.
5. Haritunians T, Mori A, O'Kelly J, Luong QT, Giles FJ and Koeffler HP: Antiproliferative activity of RAD001 (everolimus) as a single agent and combined with other agents in mantle cell lymphoma. Leukemia 21: 333-339, 2007.

6. Kumagai T, O'Kelly J, Said JW and Koeffler HP: Vitamin D2 analog 19-nor-1,25-dihydroxyvitamin D2: antitumor activity against leukemia, myeloma, and colon cancer cells. J Natl Cancer Inst 95: 896-905, 2003.

7. Hisatake J, O'Kelly J, Uskokovic MR, Tomoyasu S and Koeffler HP: Novel vitamin D(3) analog, 21-(3-methyl-3hydroxy-butyl)-19-nor D(3), that modulates cell growth, differentiation, apoptosis, cell cycle, and induction of PTEN in leukemic cells. Blood 97: 2427-2433, 2001.

8. Green DR and Reed JC: Mitochondria and apoptosis: Science 281: 1309-1312, 1998.

9. Breitman TR, Selonick SE and Collins SJ: Induction of differentiation of the human promyelocytic leukemia cell line (HL-60) by retinoic acid. Proc Natl Acad Sci USA 77: 2936-2940, 1980 . 